\title{
SHAPE OPTIMIZATION OF THERMOACOUSTIC SYSTEMS USING A 2D ADJOINT HELMHOLTZ SOLVER
}

\author{
Stefano Falco ${ }^{1, *}$, Matthew P. Juniper ${ }^{1}$ \\ ${ }^{1}$ Department of Engineering, University of Cambridge, Cambridge, United Kingdom CB21PZ
}

\begin{abstract}
Thermoacoustic instabilities, which arise due to the interaction between flames and acoustics, are sensitive to small changes to the system parameters. In this paper, we apply adjoint-based shape optimization to a $2 \mathrm{D}$ finite element Helmholtz solver to find accurately and inexpensively the shape changes that most stabilise a $2 D$ thermoacoustic system in the linear regime. We examine two cases: a Rijke tube and a turbulent swirl combustor. Both systems exhibit an unstable longitudinal mode and we suppress the instability by slightly modifying the geometry. In the case of the turbulent swirl combustor, the sensitivities are higher in the plenum and in the burner than in the combustion chamber, mainly due to the effect of the mean temperature. In the cooler regions, the local wavelength is shorter, which means that geometry changes of a given distance have more influence than they do where the local wavelength is longer. This is the first time adjoint-based shape optimization is applied to 2D Helmholtz solvers in thermoacoustics, after being previously applied to loworder thermoacoustic networks. But Helmholtz solvers have an intrinsic advantage: they can handle complex geometries. The easy scalability of this method to complex $3 D$ geometries make this tool a strong candidate for the iterative design of thermoacoustically stable combustors.
\end{abstract}

\section{Keywords:}

\section{NOMENCLATURE}

\section{Roman letters}

$c \quad$ Speed of sound [ $\left.\mathrm{m} \mathrm{s}^{-1}\right]$

$n \quad$ Interaction index $\left[\mathrm{J} \mathrm{m}^{-1}\right]$

$n \quad$ (Outward) unit normal []

$p \quad$ Pressure $\left[\mathrm{N} \mathrm{m}^{-2}\right]$

$q \quad$ Heat release per unit volume $\left[\mathrm{W} \mathrm{m}^{-3}\right.$ ]

$\boldsymbol{r}$ Reference direction []

$R \quad$ Reflection coefficient [/]

$t \quad$ Time [s]

$\boldsymbol{u} \quad$ Velocity vector $\left[\mathrm{m} \mathrm{s}^{-1}\right]$

*Corresponding author: sf620@cam.ac.uk

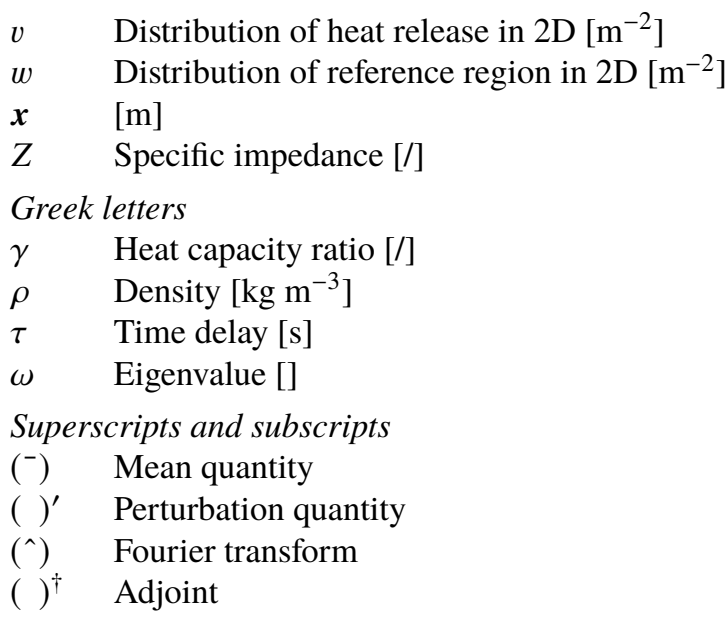

\section{INTRODUCTION}

Thermoacoustic oscillations are a major hazard to the safe performance of gas turbines and rocket engines. During the combustion process, chemical energy is converted into thermal energy. The unsteady heat release rate excites sound waves that in turn interact with the flame. If the heat release rate and the pressure perturbations are sufficiently in phase [1], then the acoustic energy increases, unless its growth is counterbalanced by the losses due to damping or acoustic radiation from the boundary [2]. These oscillations can cause mechanical fatigue, environmental noise, increased emissions and, in the worst cases, extinction of the flame or structural failure. Thermoacoustic systems are sensitive to small changes to the operating parameters or the shape of the system [3]. Even if no thermoacoustic oscillations appear when testing single components, instabilities can arise during the late stages of the design process, making it more difficult to design a stable system [4]. Ideally, gas turbines and rocket engines will be linearly stable over their operating regime. This requirement is fulfilled through extensive testing, by not operating at certain regimes, or by adding passive dampers such as Helmoltz resonators.

In network models (lumped-parameter models) [5, 6], the system is modelled as a network of ducts with an acoustically 
compact flame model. A mean flow can be included. This is the simplest approach to modelling thermoacoustic systems. In Helmholtz solvers [7], complex three-dimensional geometries can be modelled but the mean flow is set to zero. The unsteady heat release is modelled as a distributed acoustic source that is a linear function of the acoustic variables. This approach is more versatile but the size of the problem is bigger than in a network model. Lastly, large eddy simulations (LES) [8] simulate the unsteady reacting flow in the combustor with higher accuracy but considerably higher cost. This cost precludes the exclusive use of LES in the design process. For a versatile design process, all these approaches, together with experiments, need to be used jointly.

Magri and Juniper [9] applied adjoint-based sensitivity analysis in thermoacoustics for the first time. They explored both the feedback and the base state sensitivities of the eigenvalues of a time-delayed thermoacoustic system. The feedback sensitivity reveals the influence of all linear feedback mechanisms. Experiments were performed $[10,11]$ in order to test the theoretical results. This validated some results but also revealed defects in the low-order model used in [9]. The sensitivity analysis of a low-order thermoacoustic network using a wave-based approach was performed in [12], first considering a zero Mach number flow and then removing this hypothesis and adding a moving flame and a choked outlet. Adjoint methods were also used for speeding up uncertainty quantification in thermoacoustic systems: firstand second-order eigenvalue perturbations in nonlinear eigenvalue problems were used in a network model for an annular combustor [13] and in a turbulent swirl combustor [14]. The first application of adjoint equations to Helmholtz solvers appears in [15], followed by [14] for a turbulent swirl combustor and [16] for an annular combustor. Aguilar and Juniper [17] applied adjoint gradient-based optimization to a thermoacoustic network of an annular combustor to stabilize the unstable modes by tweaking the geometric parameters of the model: length and cross-sectional area of the elements. Mensah et al. [18] have recently applied a parameter-free approach to the shape sensitivity analysis of a Rijke tube using a 3D Helmholtz solver. A comprehensive review of the use of adjoints in thermoacoustic can be found in [19].

This article is structured as follows. In Section 2, following [20], we derive the relevant Helmholtz equation. In Section 3, we write the nonlinear eigenvalue problem that arises from the finite element discretization and introduce the adjoint eigenproblem. In Section 4, we derive the eigenvalue shape derivatives in Hadamard form. Finally, we apply gradient-based shape optimization to two cases of unstable thermoacoustic systems in two dimensions: an electrically heated Rijke tube in Section 5, and a turbulent swirl combustor in Section 6. By applying small geometry modifications, we stabilize both systems.

\section{THERMOACOUSTIC HELMHOLTZ EQUATION}

For a compressible inviscid flow, we consider small perturbations evolving on top of a steady zero Mach number mean flow. The flow variables are decomposed as $(\cdot)=(\bar{\cdot})+\epsilon(\cdot)^{\prime}$, with $\epsilon \ll 1$. The bar ${ }^{-}$denotes the steady mean quantity and the prime ' the unsteady perturbation quantity. For the mean quantities, we obtain $\nabla \bar{p}=0$, where $\bar{p}$ is the mean pressure. For the perturbation quantities:

$$
\begin{gathered}
\bar{\rho} \frac{\partial \boldsymbol{u}^{\prime}}{\partial t}+\nabla p^{\prime}=0 \\
\nabla \cdot\left(\frac{1}{\bar{\rho}} \nabla p^{\prime}\right)-\frac{1}{\gamma \bar{p}} \frac{\partial^{2} p^{\prime}}{\partial t^{2}}=-\frac{\gamma-1}{\gamma \bar{p}} \frac{\partial q^{\prime}}{\partial t}
\end{gathered}
$$

Equation (1) is the momentum equation and Eq. (2) is a wave equation. $\bar{\rho}$ is the mean density, $\gamma$ is the heat capacity ratio, $\boldsymbol{u}^{\prime}$ and $p^{\prime}$ are the velocity and pressure perturbation, respectively, and $q^{\prime}$ is the unsteady heat release per unit volume. The mean density, $\bar{\rho}$, contains the steady effect of the flame.

The unsteady heat release $q^{\prime}$ is modelled using a local formulation of the $n-\tau$ model [21], in which the heat release is proportional to the weighted integral of the velocity perturbation $\boldsymbol{u}^{\prime}$ over a reference region in a reference direction $\boldsymbol{r}$ at time $t$, after some time delay $\tau$.

$$
q^{\prime}(\boldsymbol{x}, t)=n v(\boldsymbol{x}) \int w(\boldsymbol{\xi}) \boldsymbol{u}^{\prime}(\boldsymbol{\xi}, t-\tau) \cdot \boldsymbol{r} \mathrm{d} \Omega
$$

$n=N \bar{Q} / \bar{u}\left(\boldsymbol{x}_{r}\right)$ is the dimensional interaction index, with units $\mathrm{J} \mathrm{m}^{-1}$, where $N$ is the non-dimensional interaction index (the magnitude of the flame transfer function), $\bar{Q}$ is the global mean heat release and $\bar{u}\left(\boldsymbol{x}_{r}\right)$ is the mean velocity at the reference point $\boldsymbol{x}_{r}$. In this paper we assume that $N$ and $\tau$ do not depend on the angular frequency. The two distributions $v$ and $w$ integrate to 1 and define the profile of the heat release rate and the reference region, respectively.

We seek separable solutions of the form:

$$
p^{\prime}(\boldsymbol{x}, t)=\operatorname{Re}\left\{\hat{p}(\boldsymbol{x}) e^{-i \omega t}\right\}
$$

After substituting $q^{\prime}$ from Eq. (3) into Eq. (2) and $\partial \boldsymbol{u}^{\prime} / \partial t$ from Eq. (1), we obtain a Helmholtz equation for the pressure perturbation with distributed heat release, acting as an acoustic source.

$$
\nabla \cdot\left(\frac{1}{\bar{\rho}} \nabla \hat{p}\right)+\frac{\omega^{2}}{\gamma \bar{p}} \hat{p}=\frac{\gamma-1}{\gamma \bar{p}} n v(\boldsymbol{x}) e^{i \omega \tau} \int w(\boldsymbol{\xi}) \frac{1}{\bar{\rho}} \nabla \hat{p} \cdot \boldsymbol{r} \mathrm{d} \Omega
$$

We impose acoustic impedance (Robin-type) boundary conditions:

$$
\frac{\partial \hat{p}}{\partial n}-\frac{i \omega}{\bar{c} Z} \hat{p}=0
$$

where $\bar{c}$ is the mean speed of sound and $Z$ is the specific impedance [22], defined as:

$$
Z=\frac{\hat{p}}{\bar{\rho} \bar{c} \hat{\boldsymbol{u}} \cdot \boldsymbol{n}}
$$

$\boldsymbol{n}$ denotes the outward unit normal. The specific impedance is in general a complex function of the frequency (and of the position). However, it can be assumed that at low frequencies $Z$ is constant. For planar acoustic waves the specific impedance is a function of the reflection coefficient $R$.

$$
Z=\frac{1+R}{1-R}
$$

The reflection coefficient is the ratio of the amplitude of the reflected wave to the amplitude of the incident wave. If $Z \rightarrow 0$ 
(open boundary), we obtain a Dirichlet (sound-soft) boundary condition for the pressure $\hat{p}=0$. If $Z \rightarrow \infty$ (closed boundary), we obtain a Neumann (sound-hard) boundary condition for the pressure $\nabla \hat{p} \cdot \boldsymbol{n}=0$. Consistently with Ref. [20], we non-dimensionalize Eqs. (5) and (6). The details are given in Appendix A.

\section{DISCRETIZATION, ADJOINT EIGENPROBLEM AND FIXED-POINT ITERATION}

The problem is discretized using the Bubnov-Galerkin finite element method, which means that the trial and the test functions belong to the same function space. We use P2 elements. The finite element approximation results in a nonlinear eigenvalue problem for the complex angular frequency $\omega$.

$$
\mathbf{N}(\omega) \mathbf{p}=\mathbf{A} \mathbf{p}+\omega \mathbf{B} \mathbf{p}+\omega^{2} \mathbf{C} \mathbf{p}-\mathbf{D}(\omega) \mathbf{p}=0
$$

The mass matrix $\mathbf{A}$ and the symmetric matrix $\mathbf{C}$ are real symmetric matrices (the Dirichlet boundary conditions are enforced symmetrically). The matrix $\mathbf{B}$ arises from the Robin boundary conditions. Unless $Z$ is purely imaginary, $\mathbf{B}$ is complex symmetric, but non-Hermitian. The unsteady heat release operator $\mathbf{D}(\omega)$ is complex and neither symmetric nor Hermitian. It depends nonlinearly on the eigenvalue $\omega$, but it is linear with respect to the eigenvector $\mathbf{p}$.

Given two complex vectors $\mathbf{u}$ and $\mathbf{v}$, we define the inner product $\langle\mathbf{v}, \mathbf{u}\rangle=\mathbf{v}^{H} \mathbf{u}$, where the superscript ${ }^{H}$ denotes the conjugate transpose of a vector or a matrix. The discrete adjoint operator $\mathbf{N}^{\dagger}$ is defined such that

$$
\left\langle\mathbf{p}^{\dagger}, \mathbf{N}(\omega) \mathbf{p}\right\rangle=\left\langle\mathbf{N}^{\dagger}\left(\omega^{*}\right) \mathbf{p}^{\dagger}, \mathbf{p}\right\rangle
$$

which is equivalent to writing

$$
\left(\mathbf{p}^{\dagger}\right)^{H} \mathbf{N}(\omega) \mathbf{p}=\left((\mathbf{N}(\omega))^{H} \mathbf{p}^{\dagger}\right)^{H} \mathbf{p}
$$

Therefore, the adjoint eigenproblem is

$$
\mathbf{N}^{\dagger}\left(\omega^{*}\right) \mathbf{p}^{\dagger}=\mathbf{A} \mathbf{p}^{\dagger}+\omega^{*} \mathbf{B}^{\mathrm{H}} \mathbf{p}^{\dagger}+\omega^{* 2} \mathbf{C}^{\dagger}-(\mathbf{D}(\omega))^{\mathrm{H}} \mathbf{p}^{\dagger}=0
$$

The spectrum of the discrete adjoint operator $\mathbf{N}^{\dagger}$ is the complex conjugate of the spectrum of $\mathbf{N}$. The adjoint eigenvector $\mathbf{p}^{\dagger}$ is the left eigenvector of $\mathbf{N}$. In the Bubnov-Galerkin finite element method, there is no difference between the discrete adjoint and the discretization of the continuous adjoint.

The nonlinear eigenvalue problems (9) and (12) are solved using a fixed-point iteration with relaxation [23, 24]. See Algorithm 1. We first set $\mathbf{D}$ to zero and solve the quadratic eigenvalue problem to find the acoustic eigenmodes. We select an eigenvalue and then start the iteration. At the $k$-th iteration we evaluate $\mathbf{D}\left(\omega^{[k]}\right)$ and solve the eigenvalue problem for $f\left(\omega^{[k]}\right)$. The new eigenvalue is

$$
\omega^{[k+1]}=g\left(\omega^{[k]} ; \alpha\right) \equiv \alpha f\left(\omega^{[k]}\right)+(1-\alpha) \omega^{[k]}
$$

where $\alpha$ is the relaxation factor. We iterate until the absolute value of the difference between the eigenvalue at two consecutive iterations is smaller than a certain tolerance. Banach's fixed-point theorem requires that $\left|g^{\prime}(\omega)\right|<1$ for the iteration to converge to

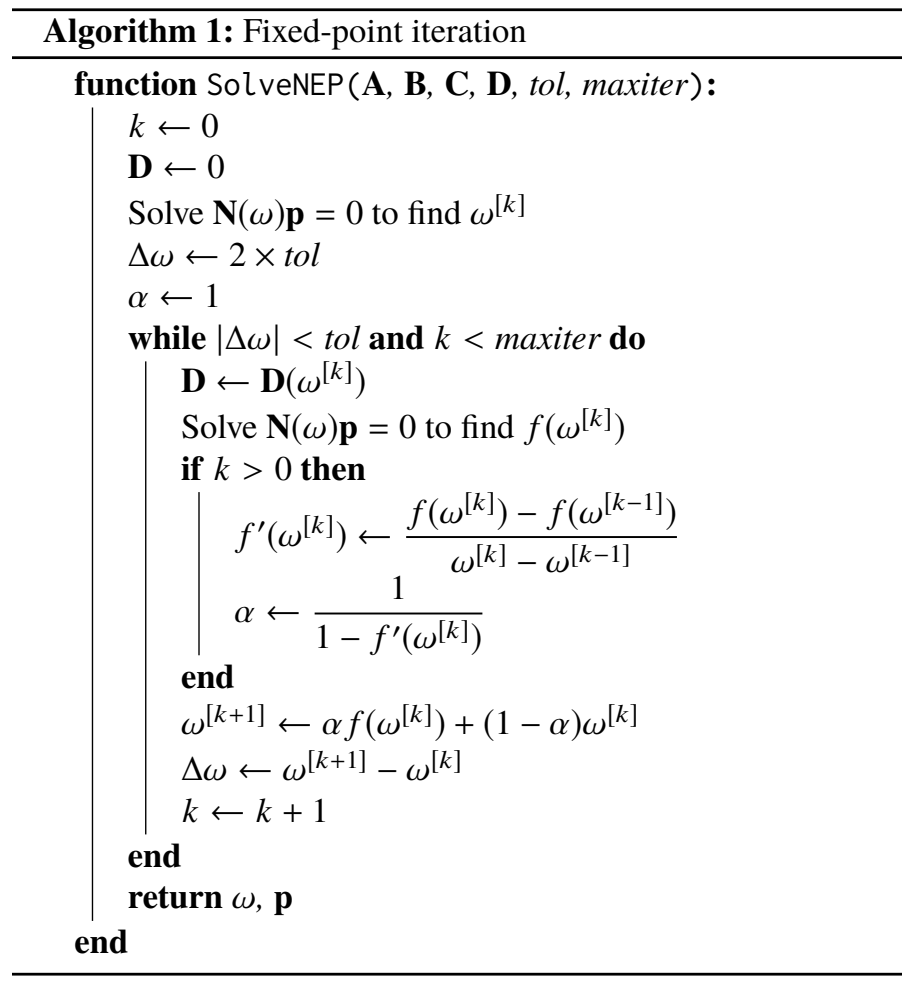

the fixed-point $\omega$. Furthermore, the rate of convergence is superlinear if $g^{\prime}(\omega)=0$, i.e. if $\alpha=1 /\left(1-f^{\prime}(\omega)\right)$. The derivative of $f$ at $\omega$ is approximated with its value at $\omega^{[k]}$ obtained using a simple backward difference.

The quadratic eigenvalue problem is solved using SLEPc [25]. The default solver for polynomial eigenvalue problems builds an Arnoldi factorization without explicitly creating the matrices. A shift-and-invert spectral transformation is used in order to enhance convergence of eigenvalues in the neighborhood of a given value. The code is written using the open source computing platform FEniCS [26, 27]. The mesh is generated by way of a Delaunay triangulation using Gmsh [28].

\section{SHAPE CALCULUS}

We are interested in how small modifications to the geometry of a combustor will affect the complex angular frequency, i.e. the eigenvalue, $\omega$, of the thermoacoustic modes. For this, we need to introduce the concept of shape derivative. Following [29], we define a one-parameter family of linear mappings

$$
T_{t}(\boldsymbol{x})=\boldsymbol{x}+t \boldsymbol{V}(\boldsymbol{x})
$$

where $t$ is a parameter and $\boldsymbol{V}$ is a continuous vector field representing the direction of the perturbation. Equation (14) is called the perturbation of identity. $T_{t}$ transforms the initial domain $\Omega$ into the deformed domain $\Omega_{t}$.

$$
\Omega_{t}=T_{t}(\Omega)
$$

We define $\mathcal{J}$ to be a shape functional, in our case an eigenvalue, $\omega$, of our problem. We define the shape derivative of $\mathcal{J}$ evaluated at $\Omega$ in the direction $\boldsymbol{V}$ as

$$
\mathrm{d} \mathcal{J}(\Omega)[\boldsymbol{V}]=\lim _{t \rightarrow 0^{+}} \frac{\mathcal{J}\left(\Omega_{t}\right)-\mathcal{J}(\Omega)}{t}
$$


The functional $\mathcal{J}$ is shape differentiable at $\Omega$ if the shape derivative (16) exists for all the directions $\mathbf{V}$ and the mapping $\mathbf{V} \mapsto \mathrm{d} \mathcal{J}(\Omega)[\boldsymbol{V}]$ is linear and continuous. In general, the shape derivative depends on the whole domain. However, if $\mathcal{J}$ is shape differentiable, under mild smoothness assumptions, its shape derivative only depends on the normal component of the vector field $\boldsymbol{V}$ at the boundary of the domain (Hadamard theorem) $[30,31]$ and can be written in the form

$$
\mathrm{d} \mathcal{J}(\Omega)[\boldsymbol{V}]=\mathrm{d} \mathcal{J}(\Gamma)[(\boldsymbol{V} \cdot \boldsymbol{n}) \boldsymbol{n}]=\int_{\Gamma}(\boldsymbol{V} \cdot \boldsymbol{n}) g \mathrm{~d} \Gamma
$$

where $g$ is the shape gradient. This result is know as Hadamard formula and the shape derivative is said to be written in Hadamard form.

\section{B-splines}

We use third-order uniform rational B-splines to parametrize the boundary. Parameter free shape optimization is possible but requires the shape changes to be smoothed in order to avoid (i) oscillations in the shape and (ii) the appearance of several local minima. We choose parametric shape optimization because it has proven easier in previous studies $[32,33]$. Third-order B-splines are smooth curves of class $C^{2}$. A B-Spline curve $\boldsymbol{B}(t)$, where $t$ is the parameter of the curve, is defined by a set of control points $\boldsymbol{P}_{0}, \ldots, \boldsymbol{P}_{n}$. By definition, the displacement field at the boundary is the derivative of the parametric curve with respect to the position of the control points.

$$
V_{i}(t)=\frac{\partial B^{j}(t)}{\partial P_{i}^{j}}
$$

The shape derivative in the direction of $\boldsymbol{V}$ becomes the shape derivative with respect to $\boldsymbol{P}_{i}$.

\section{Material and local shape derivative}

Let $\varphi_{t}$ solve some governing equations on the perturbed domain $\Omega_{t}=T_{t}(\Omega)$ and let $\boldsymbol{x}_{t} \equiv T_{t}(\boldsymbol{x})$ be a boundary point on the perturbed domain. The material shape derivative is

$$
\left.\mathrm{d} \varphi[\boldsymbol{V}](\boldsymbol{x}) \equiv \frac{\mathrm{d}}{\mathrm{d} t}\right|_{t=0} \varphi_{t}\left(\boldsymbol{x}_{t}\right)
$$

The local shape derivative is

$$
\left.\varphi^{\prime}[\boldsymbol{V}](\boldsymbol{x}) \equiv \frac{\mathrm{d}}{\mathrm{d} t}\right|_{t=0} \varphi_{t}(\boldsymbol{x})
$$

The relation between the first, which is a total derivative, and the second, which is a partial derivative is given by the chain rule

$$
\mathrm{d} \varphi[\boldsymbol{V}](\boldsymbol{x})=\varphi^{\prime}[\boldsymbol{V}](\boldsymbol{x})+\boldsymbol{V} \cdot \nabla \varphi
$$

\section{Tangential gradient and divergence}

For a function $f \in C^{2}(\Omega, \mathbb{R})$ i.e. with continuous secondorder derivatives, the tangential gradient is

$$
\nabla_{\Gamma} f \equiv \nabla f-\frac{\partial f}{\partial n} \boldsymbol{n}
$$

where $\boldsymbol{n}$ is the outward unit normal. The tangential gradient is the orthogonal projection of the gradient onto the tangent space. For a differentiable vector field $v$, the tangential divergence is

$$
\operatorname{div}_{\Gamma} \boldsymbol{v} \equiv \operatorname{div} v-(\operatorname{Dvn}) \cdot \boldsymbol{n}
$$

where Dv is the Jacobian of $\boldsymbol{v}$ and Dvn is the matrix product between $\mathrm{D} \boldsymbol{v}$ and $\boldsymbol{n}$.

\section{Shape derivatives}

Now that we have introduced the notion of shape derivative of a shape functional and the definitions of material and local shape derivative, we are ready to derive the shape derivative in Hadamard form of an eigenvalue of the thermoacoustic Helmholtz equation, Eq. (5). The shape functional is

$$
\mathcal{J}=\omega
$$

and it is subject to the constraint given by

$$
\begin{array}{rlrl}
\mathcal{N}(\omega) \hat{p}=0 & & \text { in } \Omega \\
\frac{\partial \hat{p}}{\partial n}=0 & & \text { on } \partial \Omega_{N} \\
\frac{\partial \hat{p}}{\partial n}-\frac{i \omega}{\bar{c} Z} \hat{p}=0 & & \text { on } \partial \Omega_{Z} \\
\frac{\partial \hat{p}}{\partial n} & =0 & & \text { on } \Gamma_{0}
\end{array}
$$

Equation (25) is the thermoacoustic Helmholtz equation, Eq. (5). $\partial \Omega_{N}$ is a subset of the boundary where we impose Neumann boundary conditions. $\partial \Omega_{Z}$ is a subset of the boundary where we impose Robin boundary conditions. $\Gamma_{0}$ is the deformable boundary that we wish to optimize. We take the shape derivatives of the shape functional, Eq. (24), and the constraints given by Eq. (25). In the domain, $\Omega$, and on the non-deformable boundaries, $\partial \Omega_{N}$ and $\partial \Omega_{Z}$, this is equivalent to taking the local shape derivative. Taking the material shape derivative of the Neumann boundary condition on the deformable boundary, $\Gamma_{0}$, yields the local shape derivative of the boundary condition. The material derivative of the boundary condition is identically zero, but the local derivative is not. We obtain the local shape derivative by using the chain rule and the orthogonality argument [29]. The shape derivative of Eq. (24) is

$$
\mathrm{d} \mathcal{J}[\boldsymbol{V}]=\omega^{\prime}
$$

The shape derivatives of Eq. (25) are

$$
\begin{aligned}
\frac{\partial \mathcal{N}(\omega)}{\partial \omega} \omega^{\prime} \hat{p}+\mathcal{N}(\omega) \hat{p}^{\prime} & =0 & & \text { in } \Omega \\
\frac{\partial \hat{p}^{\prime}}{\partial n} & =0 & & \text { on } \partial \Omega_{N} \\
\frac{\partial \hat{p}^{\prime}}{\partial n}-\frac{i \omega^{\prime}}{\bar{c} Z} \hat{p}-\frac{i \omega}{\bar{c} Z} \hat{p}^{\prime} & =0 & & \text { on } \partial \Omega_{Z} \\
\frac{\partial \hat{p}^{\prime}}{\partial n}+(\boldsymbol{V} \cdot \boldsymbol{n}) \frac{\partial^{2} \hat{p}}{\partial n^{2}}-\nabla_{\Gamma} \hat{p} \cdot \nabla_{\Gamma}(\boldsymbol{V} \cdot \boldsymbol{n}) & =0 & & \text { on } \Gamma_{0}
\end{aligned}
$$

Given two complex-valued square integrable functions $f$ and $g$, we define the inner product $\langle f, g\rangle \equiv \int_{\Omega} f^{*} g \mathrm{~d} \Omega$ and the product 
$\{f, g\} \equiv \int_{\partial \Omega} f^{*} g \mathrm{~d} \Gamma$. We pre-multiply (27a) by $\hat{p}^{\dagger^{*}}$, which is the complex conjugate of the adjoint pressure eigenfunction, and integrate over the domain. Then we add (26). This is equivalent to writing the shape derivative of the Lagrangian, $\mathcal{L}=\omega-$ $\left\langle\hat{p}^{\dagger}, \mathcal{N}(\omega) \hat{p}\right\rangle$, where $\hat{p}^{\dagger}$ is the Lagrange multiplier. After that, we integrate by parts to find the adjoint operator $\mathcal{N}^{\dagger}$ and the bilinear concomitant.

$$
\begin{array}{r}
\left\langle\mathcal{N}^{\dagger}\left(\omega^{*}\right) \hat{p}^{\dagger}, \hat{p}^{\prime}\right\rangle+\left\{\hat{p}^{\dagger}, \frac{1}{\bar{\rho}} \frac{\partial \hat{p}^{\prime}}{\partial n}\right\}-\left\{\frac{1}{\bar{\rho}} \frac{\partial \hat{p}^{\dagger}}{\partial n}, \hat{p}^{\prime}\right\} \\
+\left\langle\hat{p}^{\dagger}, \frac{\partial \mathcal{N}(\omega)}{\partial \omega} \hat{p}\right\rangle \omega^{\prime} \\
+\mathrm{d} \mathcal{J}[\boldsymbol{V}]=\omega^{\prime}
\end{array}
$$

The two boundary terms arise from the integration by parts of the Laplace operator. The adjoint equation is

$\nabla \cdot\left(\frac{1}{\bar{\rho}} \nabla \hat{p}^{\dagger}\right)+\frac{\omega^{* 2}}{\gamma \bar{p}} \hat{p}^{\dagger}=-\frac{\gamma-1}{\gamma \bar{p}} \nabla\left(w(\boldsymbol{x}) \frac{1}{\bar{\rho}}\right) \cdot \boldsymbol{r} \int n v(\boldsymbol{\xi}) e^{-i \omega^{*} \tau} \hat{p}^{\dagger} \mathrm{d} \Omega$

A full derivation of the adjoint equation for this particular problem can be found in [20]. We assume that the distribution of the reference region, $w$, is zero on the boundary (as recommended in [20]). It is interesting to note that the heat release region and the reference region swap. The source term is proportional to the adjoint pressure at the flame location and acts at the reference location. We can eliminate from (28) the terms containing the sensitivity $\hat{p}^{\prime}$ by choosing $\hat{p}^{\dagger}$ such that it satisfies the adjoint problem

$$
\begin{aligned}
\mathcal{N}^{\dagger}\left(\omega^{*}\right) \hat{p}^{\dagger} & =0 & & \text { on } \Omega \\
\frac{\partial \hat{p}^{\dagger}}{\partial n} & =0 & & \text { on } \partial \Omega_{N} \\
\frac{\partial \hat{p}^{\dagger}}{\partial n}+\frac{i \omega^{*}}{\bar{c} Z^{*}} \hat{p}^{\dagger} & =0 & & \text { on } \partial \Omega_{Z} \\
\frac{\partial \hat{p}^{\dagger}}{\partial n} & =0 & & \text { on } \Gamma_{0}
\end{aligned}
$$

In addition, if we group the terms containing the sensitivity $\omega^{\prime}$, we obtain a normalization condition for the adjoint eigenfunction.

$$
\left\langle\hat{p}^{\dagger}, \frac{\partial \mathcal{N}(\omega)}{\partial \omega} \hat{p}\right\rangle+\left\{\hat{p}^{\dagger}, \frac{1}{\bar{\rho}} \frac{i}{\bar{c} Z} \hat{p}\right\}_{\partial \Omega_{Z}}=1
$$

We substitute (27d) into (28) to obtain, after eliminating the sensitivity terms, an expression for the eigenvalue shape derivative.

$$
\mathrm{d} \mathcal{J}[\boldsymbol{V}]=\int_{\Gamma_{0}}(\boldsymbol{V} \cdot \boldsymbol{n})\left(\hat{p}^{\dagger^{*}} \frac{1}{\bar{\rho}} \frac{\partial^{2} \hat{p}}{\partial n^{2}}\right) \mathrm{d} \Gamma-\int_{\Gamma_{0}}\left(\hat{p}^{\dagger^{*}} \frac{1}{\bar{\rho}} \nabla_{\Gamma} \hat{p}\right) \cdot \nabla_{\Gamma}(\boldsymbol{V} \cdot \boldsymbol{n}) \mathrm{d} \Gamma
$$

The first integral is in Hadamard form; the second is not but can be made so by applying the tangential Stokes formula [31]

$$
\int_{\Gamma} g \operatorname{div}_{\Gamma} \boldsymbol{v}+\nabla_{\Gamma} g \cdot \boldsymbol{v} \mathrm{d} \Gamma=\int_{\Gamma} \kappa g \boldsymbol{v} \cdot \boldsymbol{n} \mathrm{d} \Gamma
$$

where $g$ is a real-valued function, $\boldsymbol{v}$ is a vector-valued function and $\kappa$ is the curvature: $g=\boldsymbol{V} \cdot \boldsymbol{n}$ and $\boldsymbol{v}=\hat{p}^{\hat{\dagger}^{*}} \frac{1}{\bar{\rho}} \nabla_{\Gamma} \hat{p}$. The eigenvalue shape derivative in Hadamard form is

$$
\begin{aligned}
& \mathrm{d} \mathcal{J}[\boldsymbol{V}]=\int_{\Gamma_{0}}(\boldsymbol{V} \cdot \boldsymbol{n})\left(\hat{p}^{\hat{\dagger}^{*}} \frac{1}{\bar{\rho}} \frac{\partial^{2} \hat{p}}{\partial n^{2}}\right) \mathrm{d} \Gamma \\
& -\int_{\Gamma_{0}}(\boldsymbol{V} \cdot \boldsymbol{n})\left[\kappa\left(\hat{p}^{\hat{\dagger}^{*}} \frac{1}{\bar{\rho}} \nabla_{\Gamma} \hat{p}\right) \cdot \boldsymbol{n}-\operatorname{div}_{\Gamma}\left(\hat{p}^{\dagger^{*}} \frac{1}{\bar{\rho}} \nabla_{\Gamma} \hat{p}\right)\right] \mathrm{d} \Gamma
\end{aligned}
$$

In order to evaluate (34), we need the direct eigenfunction, $\hat{p}$, the adjoint eigenfunction, $\hat{p}^{\dagger}$, the boundary displacements, $\boldsymbol{V}$, and the curvature $\kappa$. The last two depend on the shape parametrization.

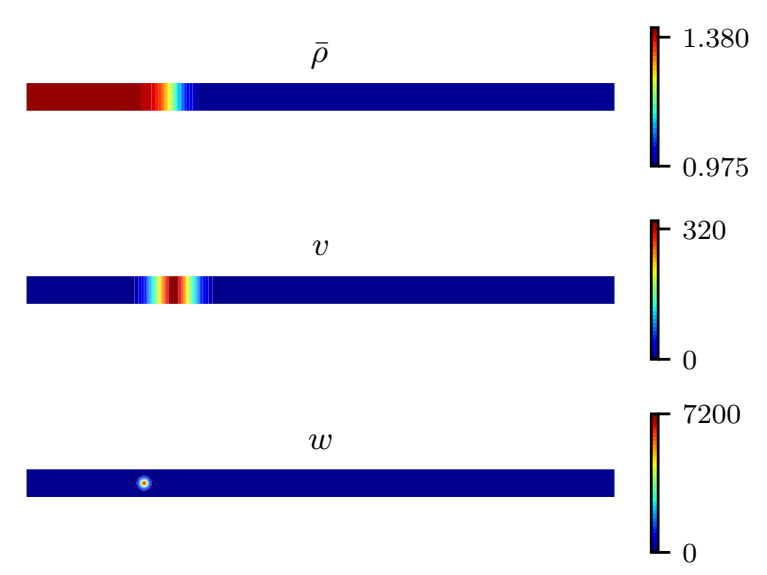

FIGURE 1: Nondimensional mean density, $\bar{\rho}$, distribution of heat release, $v$, and distribution of reference region, $w$, in the two-dimensional model of the electrically heated Rijke tube.
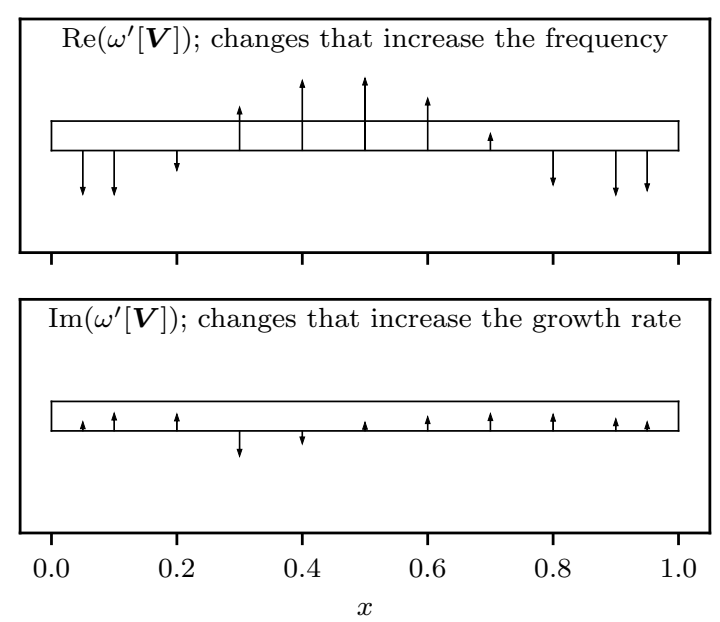

FIGURE 2: Real (top) and imaginary (bottom) components of the eigenvalue shape derivatives for each control point at the bottom boundary of the Rijke tube. The real part gives the influence on the frequency and the imaginary part gives the influence on the growth rate.

\section{RIJKE TUBE}

The first case we consider is a two-dimensional model of an electrically heated Rijke tube $[10,20]$. Acoustic impedance boundary conditions are imposed both at the inlet and at the outlet 
boundary. Sound-hard boundary conditions are imposed at the radial wall. $x_{f}$ denotes the position of the hot wire and $a_{f}$ is the standard deviation of the heat release distribution, which is homogeneous in the radial direction. $d_{f}$ is the diameter of the duct at the heat source. $x_{r}$ denotes the centre of the reference region and $a_{r}$ is the standard deviation of the reference region distribution. The standard deviation is the same in the longitudinal and in the radial direction. $\rho_{u}$ and $\rho_{d}$ denote the mean density in the cold and in the hot region, respectively. All the parameters can be found in Table I of [20], apart from $a_{r}$, which here is $4.7 \times 10^{-3} \mathrm{~m}$. The mean density, which is homogeneous in the radial direction, is

$$
\bar{\rho}(x, r)=\rho_{d}+\frac{\rho_{d}-\rho_{u}}{2}\left[1+\tanh \left(\frac{x-x_{f}}{a_{f}}\right)\right]
$$

The distribution of the heat release across the hot wire is

$$
v(x, r)=\frac{1}{a_{f} d_{f} \sqrt{2 \pi}} \exp \left(-\frac{\left(x-x_{f}\right)^{2}}{2 a_{f}^{2}}\right)
$$

The distribution of the reference region is

$$
w(x, r)=\frac{1}{a_{r}^{2} 2 \pi} \exp \left(-\frac{\left(x-x_{r}\right)^{2}+r^{2}}{2 a_{r}^{2}}\right)
$$

$w$ is a Gaussian function. It vanishes at infinity but it does not have compact support. However, its standard deviation, $a_{r}$, is small and we restrict $w$ so that it is zero at the boundary.

Figure 1 shows the mean density $\bar{\rho}$, and the distributions of heat release and reference region, $v$ and $w$ for the two-dimensional model of the Rijke tube. In two dimensions, the interaction index of the local $n-\tau$ model is $n=\pi / 4 d_{f} N \bar{Q} / \bar{u}\left(\boldsymbol{x}_{r}\right)$.

Defining two sets of control points, we parametrize the bottom and top boundaries using $3^{\text {rd }}$ order uniform rational Bsplines. We fix the inlet and outlet boundaries. We compute the system's first direct and adjoint thermoacoustic eigenmode, which is unstable. Then, using (34), we find the shape derivatives for each control point. The real part gives the direction of greatest positive change of the angular frequency of the eigenmode. The imaginary part gives the direction of greatest positive change of the growth rate of the eigenmode. Therefore, we move the control points in the opposite direction of the imaginary part of the shape derivative. This reduces the growth rate and eventually makes the mode stable. In Fig. 2 we show the shape derivatives for each control point as vectors. As expected, they are all orthogonal to the boundary. A tangential displacement would only result in a reparametrization of the boundary, without changing its shape or affecting the eigenvalue. The eigenmode becomes stable after 3 iterations. We continue to reduce the growth rate in order to accentuate the deformation so that the differences between the two shapes and between the eigenfunctions are more visible. Fig. 3 shows the initial and the deformed boundary (after 10 iterations). Fig. 4 shows the modulus of the pressure eigenfunction before and after the optimization. This shape change reduces the longitudinal component of the pressure gradient in the reference region. In the $n-\tau$ model, the heat release rate fluctuation is proportional to the the velocity perturbation in the reference region.
Therefore, this shape change reduces the growth rate by reducing the amplitude of the acoustic velocity in the reference region.

The results for the initial domain match those in the onedimensional model of Ref. [20] and against the finite volume Helmholtz solver AVSP [7].

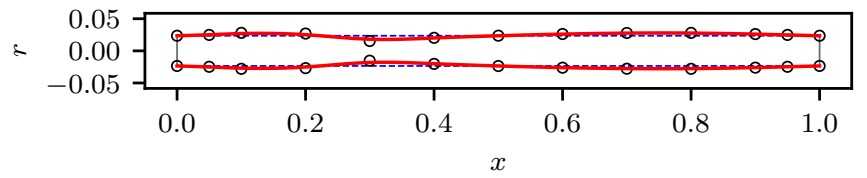

FIGURE 3: Initial (- -) and deformed (-) boundary of the Rijke tube after 10 iterations. The circles represent the control points of the deformed boundary. The control points at the inlet and at the outlet are fixed.

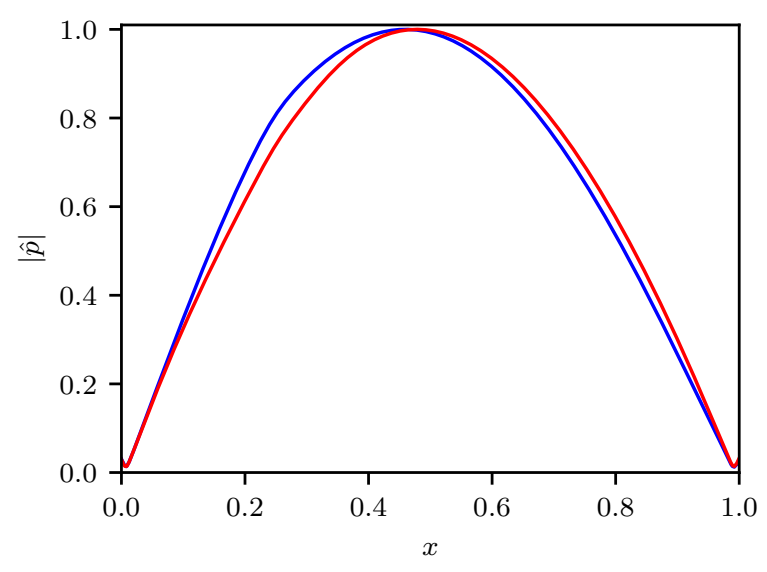

FIGURE 4: Absolute value of the direct pressure eigenfunction $\hat{p}$ relative to the first eigenmode of the electrically heated Rijke tube. (-) initial domain, (-) deformed domain after 10 iterations. The associated nondimensional eigenvalues are $\omega_{\Omega}=3.539521+i 0.001984$ and $\omega_{\Omega_{t}}=$ $3.688497-i 0.009876$, respectively.

\section{TURBULENT SWIRL COMBUSTOR}

The turbulent swirl combustor investigated here is that of EM2C laboratory and has been extensively studied in the context of combustion instabilities [14, 34-37]. It consists of a cylindrical plenum, a convergent duct, a swirler, and a cylindrical combustion chamber. A mixture of methane and air at an equivalence ratio of 0.8 is injected upstream of the plenum. The total power of the flame at the operating point considered in this study is $3.03 \mathrm{~kW}$. The parameters of the model, taken from [37], are reported in Table 1. This system exhibits an unstable longitudinal mode.

The eigenvalue we found with our 2D planar code is $181+$ $i 3.33 \mathrm{~s}^{-1}$. We calculated the same case in 2D with AVSP and found a complex angular frequency of $182+i 3.28 \mathrm{~s}^{-1}$.

Acoustic impedance boundary conditions are imposed at the outlet boundary. Sound-hard boundary conditions are imposed at the walls. Figure 5 shows the mean density $\bar{\rho}$, and the distributions of heat release and reference region, $v$ and $w$. Their analytical expression are given by Eqs. (35), (36) and (37), respectively. 
TABLE 1: Turbulent swirl combustor

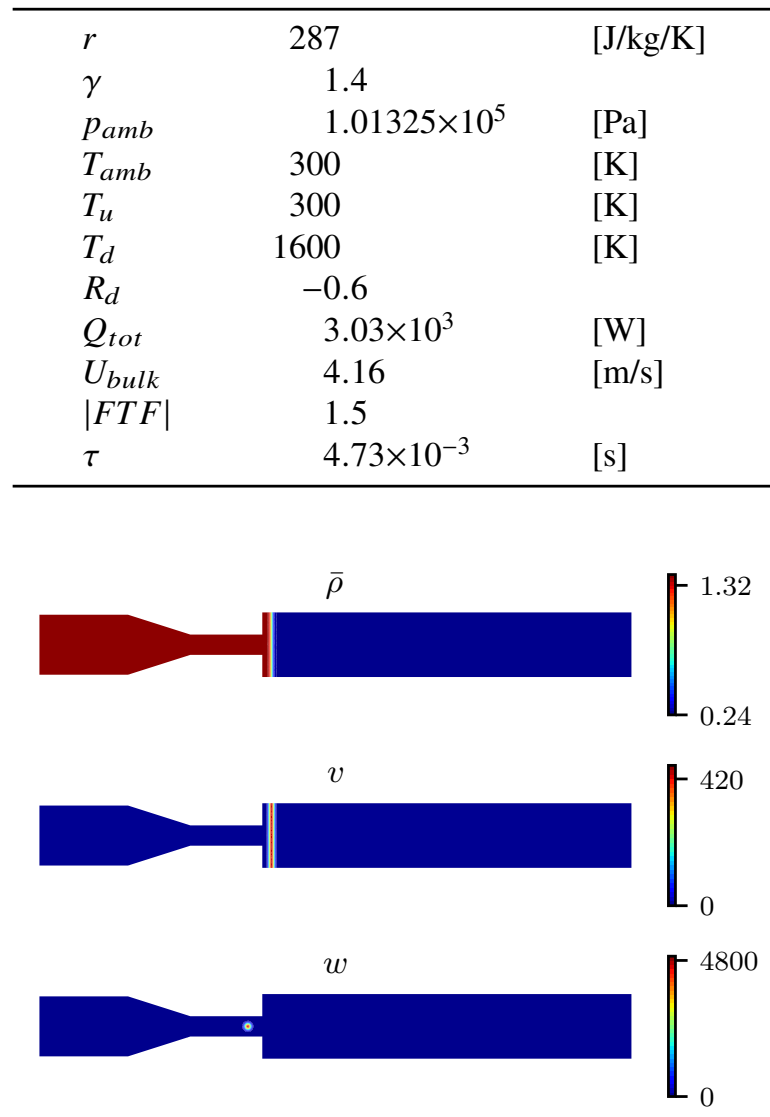

FIGURE 5: Nondimensional mean density, $\bar{\rho}$, distribution of heat release, $v$, and distribution of reference region, $w$, in the two-dimensional model of the turbulent swirl combustor.

We parametrize the entire geometry except for the outlet, which is an open boundary, including more control points where there are sharp corners. We compute the direct and the adjoint eigenmode and apply Eq. (34) to find the shape derivatives. Figure 6 shows the eigenvalue shape derivatives for each control point. The eigenvalue is more sensitive in the plenum and much more sensitive in the burner than in the combustion chamber. This is because the gas is cooler upstream of the temperature jump across the flame so the local wavelength is shorter and therefore geometry modifications have more influence. The shape derivatives show that reducing the size of the plenum and increasing the cross sectional area of the burner in the reference region reduces the growth rate. In any case, manufacturers are reluctant to change the geometry of the burner and the combustion chamber because they are determined by other considerations, but they are willing to modify the geometry of the plenum.

We fix the geometry of both the burner and the combustion chamber and allow the geometry of the plenum to vary. The unstable mode is stabilized after 5 iterations. In Fig. 7 we show the initial and the final shape of the plenum. In the final shape, the control points are moved inwards. We show in Fig. 8 the direct and adjoint eigenfunctions for the initial and deformed domain. We see again that the pressure gradient, and so the velocity
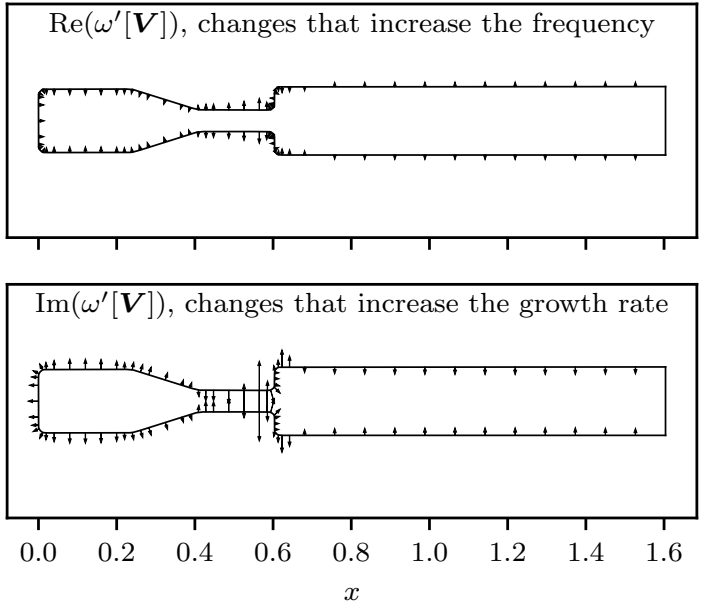

FIGURE 6: Real (top) and imaginary (bottom) components of the eigenvalue shape derivatives for each control point at the deformable boundary of the turbulent swirl combustor. The real part gives the influence on the frequency and the imaginary part gives the influence on the growth rate.

perturbation, has reduced in the reference location resulting in smaller heat release rate fluctuations. This shows how to stabilize a thermoacoustic mode while keeping the flame transfer function constant.

\section{CONCLUSION}

We have implemented a direct and adjoint thermoacoustic Helmoltz solver using 2D finite elements. We express the shape derivative of the eigenvalue in Hadamard form, i.e. for arbitraty shape changes. Using B-splines we then parametrize the shapes of two thermoacoustic systems found in the literature. We use the Hadamard expression to calculate the derivative of the eigenvalues with respect to the $\mathrm{B}$-spline parameters and interpret these results physically. We then use gradient-based optimization to stabilize both systems by making small geometry modifications in the most influential regions.

This study shows how to implement shape optimization with an adjoint thermoacoustic Helmholtz solver in 2D. It exploits the fact that thermoacoustic systems are sensitive to small modifications [3] and shows that models of unsteady systems available in the literature can be stabilized with small geometry changes.

Direct and adjoint thermoacoustic Helmholtz solvers can easily be extended to 3D, albeit at increased computational cost. The advantage of using FEniCS with SLEPc is that parallelization is handled in the background and that numerical analysis researchers continue to develop and implement increasingly powerful algorithms.

The implementation in this paper assumes that the flame response is unaffected by the geometry changes. This is reasonable only for geometry changes in the plenum. If geometry changes were to be considered in practice, then a more realistic model of the flame response would need to be included. This would be a flame transfer function with distributed time delays with different time delays for the different geometries. The optimization method described in this paper might potentially be applied to the 


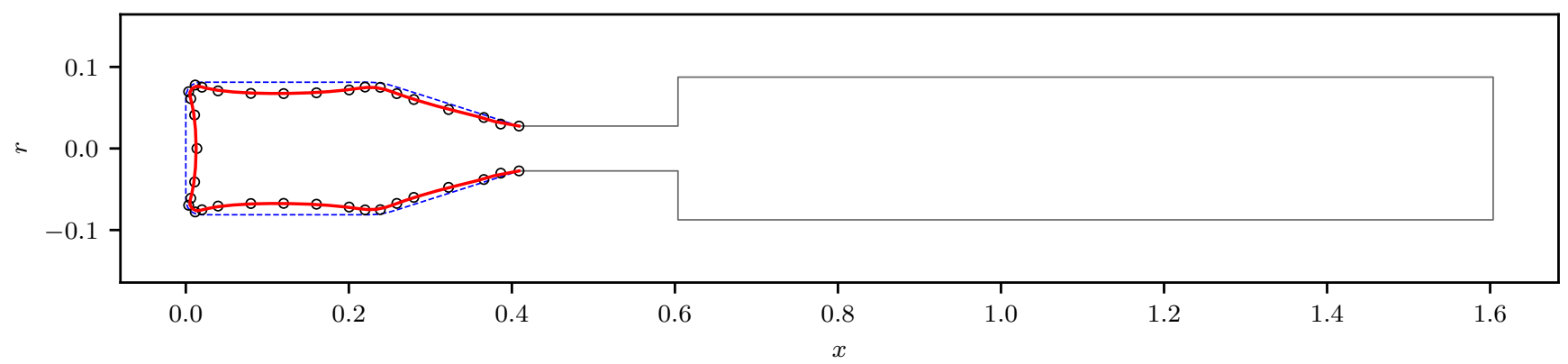

FIGURE 7: Initial (- -) and deformed (-) boundary of the turbulent swirl combustor after gradient-based optimization has been used to stabilize the device by changing the plenum while fixing the burner and the combustion chamber. The lengths are non-dimensionalized with respect to the acoustic wavelength of the combustion chamber.
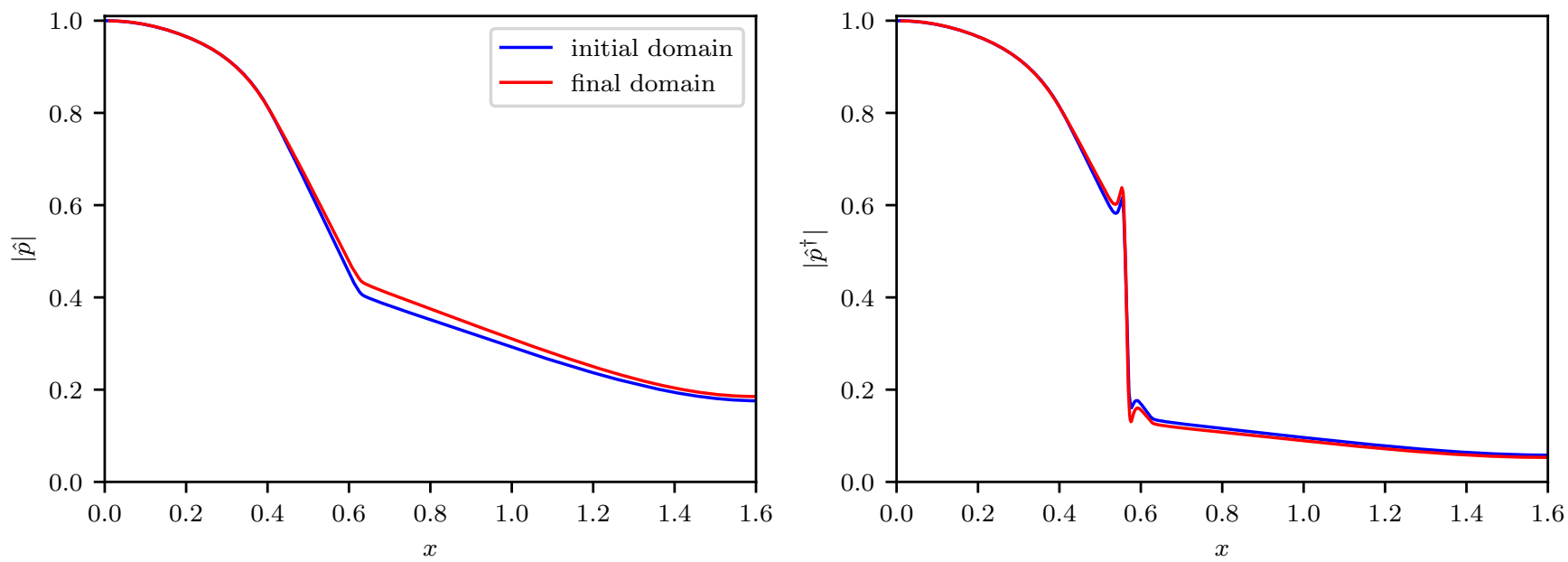

FIGURE 8: (Left) Modulus of the direct pressure eigenfunction $\hat{p}$ relative to the first eigenmode of the turbulent swirl combustor. ( - ) initial domain, $\Omega,(-)$ deformed domain, $\Omega_{t}$. The associated direct nondimensional eigenvalues are $\omega_{\Omega}=1.3157+i 0.0241$ and $\omega_{\Omega_{t}}=1.3644-i 0.0978$, respectively. (Right) Modulus of the adjoint pressure eigenfunction $\hat{p}^{\dagger}$ relative to the first eigenmode of the turbulent swirl combustor. (一) initial domain, $\Omega$, (一) deformed domain, $\Omega_{t}$. The source acts locally in the reference region. The wiggles are due to the fact that the source is proportional to the component of the gradient in the $x$-direction of the distribution of reference region, $w$, which is a Gaussian function.

linearized reactive flow solver presented in [38], where the flame transfer function is not needed a priori.

The following issues will need to be addressed if this method is applied to more realistic configurations such as annular combustors. For semi-simple eigenvalues the problem is not totally differentiable with respect to all the parameters. A famous example is the buckling of columns [31]. Issues for degenerate eigenvalues arise if the eigenvalue loses degeneracy due to a parameter change. For defective eigenvalues, the so-called exceptional points, the sensitivity is infinite. However, exceptional points are very rare.

In conclusion, this paper shows that shape optimization of thermoacoustic Helmholtz solvers is possible and that it would be a useful design tool in the gas turbine industry.

\section{ACKNOWLEDGEMENT}

We gratefully acknowledge the European Union's Framework Programme for Research and Innovation Horizon 2020 under the Marie Skłodowska Curie grant agreement ANNULIGhT
No 765998 for funding this project.

\section{REFERENCES}

[1] Rayleigh, Lord. "The Expalnation of Certain Acoustic Phenomena." Nature Vol. 18, No. 455 (1878): pp. 319321. DOI 10.1038/018319a0. URL http://www.nature.com/ articles/018319a0.

[2] Chu, Boa-Teh. "On the energy transfer to small disturbances in fluid flow (Part I)." Acta Mech. Vol. 1, No. 3 (1965): pp. 215-234. DOI 10.1007/BF01387235. URL http://link. springer.com/10.1007/BF01387235.

[3] Juniper, Matthew P. and Sujith, Raman I. "Sensitivity and Nonlinearity of Thermoacoustic Oscillations." Annu. Rev. Fluid Mech. Vol. 50, No. 1 (2018): pp. 661-689. DOI 10.1146/annurev-fluid-122316045125. URL http://www.annualreviews.org/doi/10.1146/ annurev-fluid-122316-045125.

[4] Mongia, H. C., Held, T. J., Hsiao, G. C. and Pandalai, R. P. "Challenges and Progress in Controlling Dynamics 
in Gas Turbine Combustors." J. Propuls. Power Vol. 19, No. 5 (2003): pp. 822-829. DOI 10.2514/2.6197. URL https://arc.aiaa.org/doi/10.2514/2.6197.

[5] Schuermans, Bruno B. H., Polifke, Wolfgang and Paschereit, Christian Oliver. "Modeling Transfer Matrices of Premixed Flames and Comparison With Experimental Results." Proc. ASME 1999 Int. Gas Turbine Aeroengine Congr. Exhib. Vol. 2 Coal, Biomass Altern. Fuels; Combust. Fuels; Oil Gas Appl. Cycle Innov. 1999. DOI 10.1115/99-GT-132. URL https://asmedigitalcollection.asme.org/GT/proceedings/ GT1999/78590/Indianapolis, Indiana,USA/248306.

[6] Dowling, Ann P. and Stow, Simon R. "Acoustic Analysis of Gas Turbine Combustors." J. Propuls. Power Vol. 19, No. 5 (2003): pp. 751-764. DOI 10.2514/2.6192. URL http://arc.aiaa.org/doi/10.2514/2.6192.

[7] Nicoud, Franck, Benoit, Laurent, Sensiau, Claude and Poinsot, Thierry. "Acoustic Modes in Combustors with Complex Impedances and Multidimensional Active Flames." AIAA J. Vol. 45, No. 2 (2007): pp. 426-441. DOI 10.2514/1.24933. URL https://arc.aiaa.org/doi/10.2514/1. 24933.

[8] Poinsot, Thierry. "Prediction and control of combustion instabilities in real engines." Proc. Combust. Inst. Vol. 36, No. 1 (2017): pp. 1-28. DOI 10.1016/j.proci.2016.05.007. URL http://dx.doi.org/10.1016/j.proci.2016.05.007.

[9] Magri, Luca and Juniper, Matthew P. "Sensitivity analysis of a time-delayed thermo-acoustic system via an adjoint-based approach." J. Fluid Mech. Vol. 719 (2013): pp. 183-202. DOI 10.1017/jfm.2012.639. URL https://www.cambridge.org/core/product/identifier/ S0022112012006398/type/journal\{_\}article.

[10] Rigas, Georgios, Jamieson, Nicholas P., Li, Larry K. B. and Juniper, Matthew P. "Experimental sensitivity analysis and control of thermoacoustic systems." J. Fluid Mech. Vol. 787 (2016): p. R1. DOI 10.1017/jfm.2015.715. URL https://www.cambridge.org/core/product/identifier/ S0022112015007156/type/journal \{_\}article.

[11] Jamieson, Nicholas P., Rigas, Georgios and Juniper, Matthew P. "Experimental sensitivity analysis via a secondary heat source in an oscillating thermoacoustic system.” Int. J. Spray Combust. Dyn. Vol. 9, No. 4 (2017): pp. 230-240. DOI 10.1177/1756827717696325. URL http: //journals.sagepub.com/doi/10.1177/1756827717696325.

[12] Aguilar, José G., Magri, Luca and Juniper, Matthew P. "Adjoint-based sensitivity analysis of low-order thermoacoustic networks using a wave-based approach." $J$. Comput. Phys. Vol. 341 (2017): pp. 163-181. DOI 10.1016/j.jcp.2017.04.013. URL http://dx.doi.org/10.1016/ j.jcp.2017.04.013.

[13] Magri, Luca, Bauerheim, Michael, Nicoud, Franck and Juniper, Matthew P. "Stability analysis of thermo-acoustic nonlinear eigenproblems in annular combustors. Part II. Uncertainty quantification.” J. Comput. Phys. Vol. 325 (2016): pp. 411-421. DOI 10.1016/j.jcp.2016.08.043. URL http://dx.doi.org/10.1016/j.jcp.2016.08.043.
[14] Silva, Camilo F., Magri, Luca, Runte, Thomas and Polifke, Wolfgang. "Uncertainty Quantification of Growth Rates of Thermoacoustic Instability by an Adjoint Helmholtz Solver.” J. Eng. Gas Turbines Power Vol. 139, No. 1 (2017): p. 011901. DOI 10.1115/1.4034203. URL https://asmedigitalcollection.asme.org/ gasturbinespower/article/doi/10.1115/1.4034203/374361/ Uncertainty-Quantification-of-Growth-Rates-of.

[15] Juniper, Matthew. P., Magri, Luca, Bauerheim, Michael and Nicoud, Franck. "Sensitivity analysis of thermoacoustic eigenproblems with adjoint methods." Proc. Cent. Turbul. Res. Summer Progr. (2014): pp. 189-198URL https://web.stanford.edu/group/ctr/Summer/ SP14/06\{_\}Combustion/09\{_\}juniper.pdf.

[16] Mensah, Georg A. and Moeck, Jonas P. "Acoustic Damper Placement and Tuning for Annular Combustors: An Adjoint-Based Optimization Study." J. Eng. Gas Turbines Power Vol. 139, No. 6 (2017): p. 061501. DOI 10.1115/1.4035201. URL https://asmedigitalcollection.asme.org/ gasturbinespower/article/doi/10.1115/1.4035201/374005/ Acoustic-Damper-Placement-and-Tuning-for-Annular.

[17] Aguilar, José G. and Juniper, Matthew P. "Adjoint Methods for Elimination of Thermoacoustic Oscillations in a Model Annular Combustor via Small Geometry Modifications." Proc. ASME Turbo Expo 2018 Turbomach. Tech. Conf. Expo. Vol. 4A Combust. Fuels, Emiss.: pp. 1-11. 2018. DOI 10.1115/GT201875692. URL https://asmedigitalcollection.asme.org/GT/ proceedings/GT2018/51050/Oslo,Norway/271106.

[18] Mensah, Georg A, Orchini, Alessandro and Moeck, Jonas P. "Adjoint-based computation of shape sensitivity in a Rijketube." Proc. 23rd Int. Congr. Acoust. 1: pp. 54895496. 2019. URL http://pub.dega-akustik.de/ICA2019/ data/articles/000816.pdf.

[19] Magri, Luca. "Adjoint Methods as Design Tools in Thermoacoustics." Appl. Mech. Rev. Vol. 71, No. 2 (2019): p. 020801. DOI 10.1115/1.4042821. URL https:// asmedigitalcollection.asme.org/appliedmechanicsreviews/ article/doi/10.1115/1.4042821/726671/

Adjoint-Methods-as-Design-Tools-in-Thermoacoustics.

[20] Juniper, Matthew P. "Sensitivity analysis of thermoacoustic instability with adjoint Helmholtz solvers." Phys. Rev. Fluids Vol. 3, No. 11 (2018): p. 110509. DOI 10.1103/PhysRevFluids.3.110509. URL https://link.aps.org/doi/10.1103/ PhysRevFluids.3.110509.

[21] Crocco, Luigi. "Aspects of Combustion Stability in Liquid Propellant Rocket Motors Part I: Fundamentals. Low Frequency Instability With Monopropellants." J. Am. Rocket Soc. Vol. 21, No. 6 (1951): pp. 163-178. DOI 10.2514/8.4393. URL https://arc.aiaa.org/doi/10.2514/8. 4393.

[22] Rienstra, Sjoerd W. and Hirschberg, Avraham. An Introduction to Acoustics. Eindhoven University of Technology (2004). URL https://www.win.tue.nl/\{ $\}$ sjoerdr/ papers/boek.pdf. 
[23] Ni, Franchine. "Accounting for complex flow-acoustic interactions in a 3D thermo-acoustic Helmholtz solver." Ph.D. thesis, Institut National Polytechnique de Toulouse, Toulouse, France. 2017.

[24] Mensah, Georg Atta. "Efficient Computation of Thermoacoustic Modes.” Ph.D. thesis, Technische Universität Berlin, Berlin, Germany. 2018.

[25] Hernandez, Vicente, Roman, Jose E. and Vidal, Vicente. "SLEPc: A Scalable and Flexible Toolkit for the Solution of Eig." ACM Trans. Math. Softw. Vol. 31, No. 3 (2005): pp. 351-362. DOI 10.1145/1089014.1089019. URL http: //portal.acm.org/citation.cfm?doid=1089014.1089019.

[26] Alnæs, Martin S., Blechta, Jan, Hake, Johan, Johansson, August, Kehlet, Benjamin, Logg, Anders, Richardson, Chris, Ring, Johannes, Rognes, Marie E. and Wells, Garth N. "The FEniCS Project Version 1.5." Archive of Numerical Software Vol. 3, No. 100. DOI 10.11588/ans.2015.100.20553.

[27] Logg, Anders, Mardal, Kent-Andre, Wells, Garth N. et al. Automated Solution of Differential Equations by the Finite Element Method. Springer (2012). DOI 10.1007/978-3642-23099-8.

[28] Geuzaine, Christophe and Remacle, Jean-François. "Gmsh: A 3-D finite element mesh generator with built-in preand post-processing facilities." Int. J. Numer. Methods Eng. Vol. 79, No. 11 (2009): pp. 1309-1331. DOI 10.1002/nme.2579. URL http://doi.wiley.com/10.1002/ nme. 2579 .

[29] Schmidt, Stephan and Schulz, Volker. "Shape derivatives for general objective functions and the incompressible NavierStokes equations." Control Cybern. Vol. 39, No. 3 (2010): pp. 677-713.

[30] Sokolowski, Jan and Zolesio, Jean-Paul. Introduction to Shape Optimization. Vol. 16 of Springer Series in Computational Mathematics. Springer Berlin Heidelberg, Berlin, Heidelberg (1992). DOI 10.1007/978-3-642-58106-9. URL http://link.springer.com/10.1007/978-3-642-58106-9.

[31] Delfour, Michel. C. and Zolésio, Jean-Paul. Shapes and Geometries: Metrics, Analysis, Differential Calculus, and Optimization, Second Edition. Society for Industrial and Applied Mathematics, Philadelphia (2011). DOI 10.1137/1.9780898719826. URL http://epubs.siam. org/doi/book/10.1137/1.9780898719826.

[32] Kungurtsev, Petr V. and Juniper, Matthew P. "Adjointbased shape optimization of the microchannels in an inkjet printhead." J. Fluid Mech. Vol. 871 (2019): pp. 113-138. DOI 10.1017/jfm.2019.271. URL https://www.cambridge.org/core/product/identifier/ S0022112019002714/type/journal___\}article.

[33] Brewster, Jack and Juniper, Matthew P. "Shape sensitivity of eigenvalues in hydrodynamic stability, with physical interpretation for the flow around a cylinder." Eur. J. Mech. - B/Fluids Vol. 80 (2020): pp. 80-91. DOI 10.1016/j.euromechflu.2019.11.007. URL https:// linkinghub.elsevier.com/retrieve/pii/S0997754619300962.
[34] Palies, Paul, Durox, Daniel, Schuller, Thierry and Candel, Sébastien. "The combined dynamics of swirler and turbulent premixed swirling flames." Combust. Flame Vol. 157, No. 9 (2010): pp. 1698-1717. DOI 10.1016/j.combustflame.2010.02.011. URL http://dx.doi. org/10.1016/j.combustflame.2010.02.011.

[35] Palies, Paul, Durox, Daniel, Schuller, Thierry and Candel, Sébastien. "Nonlinear combustion instability analysis based on the flame describing function applied to turbulent premixed swirling flames." Combust. Flame Vol. 158, No. 10 (2011): pp. 1980-1991. DOI 10.1016/j.combustflame.2011.02.012. URL http://dx.doi. org/10.1016/j.combustflame.2011.02.012.

[36] Silva, Camilo F., Nicoud, Franck, Schuller, Thierry, Durox, Daniel and Candel, Sebastien. "Combining a Helmholtz solver with the flame describing function to assess combustion instability in a premixed swirled combustor." Combust. Flame Vol. 160, No. 9 (2013): pp. 1743-1754. DOI 10.1016/j.combustflame.2013.03.020. URL http://dx.doi. org/10.1016/j.combustflame.2013.03.020.

[37] Avdonin, Alexander, Jaensch, Stefan, Silva, Camilo F., Češnovar, Matic and Polifke, Wolfgang. "Uncertainty quantification and sensitivity analysis of thermoacoustic stability with non-intrusive polynomial chaos expansion." Combust. Flame Vol. 189 (2018): pp. 300-310. DOI 10.1016/j.combustflame.2017.11.001. URL https:// linkinghub.elsevier.com/retrieve/pii/S0010218017304388.

[38] Avdonin, Alexander, Meindl, Max and Polifke, Wolfgang. "Thermoacoustic analysis of a laminar premixed flame using a linearized reactive flow solver." Proc. Combust. Inst. Vol. 37, No. 4 (2019): pp. 5307-5314. DOI 10.1016/j.proci.2018.06.142. URL https://linkinghub. elsevier.com/retrieve/pii/S1540748918303250.

\section{APPENDIX A.}

The reference length, $L$, is the acoustic wavelength of the tube, in the case of the Rijke tube, or the acoustic wavelength of the combustion chamber, in the case of the turbulent swirl combustor. The reference time is $L / c_{a}$, where $c_{a}=\sqrt{\gamma p_{a} / \rho_{a}}$ is the speed of sound in the ambient fluid and $\rho_{a}$ and $p_{a}$ are the ambient density and pressure, respectively. We denote the non-dimensional quantities by the tilde . The independent and dependent variables are non-dimensionalized as follows

$$
x=\tilde{x} L, \quad t=\tilde{t} \frac{L}{c_{a}}, \quad \rho=\tilde{\rho} \frac{\rho_{a}}{\gamma}, \quad \boldsymbol{u}=\tilde{\boldsymbol{u}} c_{a}, \quad p=\tilde{p} p_{a}
$$

The non-dimensionalization is the same for the mean quantities and for the perturbation quantities. The non-dimensional ambient density equals $\gamma$. Since the mean pressure is constant, the nondimensional mean pressure equals 1 everywhere. The parameters of the $n-\tau$ model are scaled as follows. The local interaction index is scaled as $n=\tilde{n} p_{a} L$ in two dimensions. The time delay $\tau$ is scaled as $\tau=\tilde{\tau} L / c_{a}$. The complex angular frequency $\omega$ is scaled as $\omega=\tilde{\omega} c_{a} / L$. 